\title{
Perfil dos portadores de diabetes mellitus atendidos em farmácias particulares de Sergipe, Brasil
}

\author{
Profile of carriers of diabetes mellitus served in private pharmacies, Sergipe, Brazil \\ C. O. Gois ${ }^{1}$; S. V. M. A. Lima ${ }^{1}$; A. C. F. S. Santos ${ }^{1} ;$ H. N. Melo ${ }^{2}$ \\ ${ }^{1}$ Departamento de Enfermagem, Universidade Federal de Sergipe, 49400-000, Lagarto-Sergipe, Brasil \\ 2 Programa de Pós-Graduação em Ciências da Saúde, Universidade Federal de Sergipe, 49100-000, Aracaju - \\ Sergipe, Brasil \\ *carla_gois1993@hotmail.com
}

(Recebido em 30 de maio de 2017; aceito em 04 de novembro de 2017)

\begin{abstract}
O Diabetes Mellitus é uma doença crônica, caracterizada por um desequilíbrio glicêmico resultante do defeito na ação/secreção da insulina. O objetivo desse estudo foi conhecer o perfil dos portadores de Diabetes Mellitus que procuram drogarias particulares para obtenção de medicamentos e acompanhamento do agravo. Trata-se de um estudo transversal realizado em duas farmácias particulares no município de Itabaiana/Sergipe, no qual foi aplicado um formulário com 10 questões a respeito de dados socioeconômicos e sobre o Diabetes Mellitus. Foram analisados 90 formulários. O resultado obtido contou com predominância do sexo feminino, baixa renda familiar, com baixo grau de escolaridade, que desconhecem o tipo de Diabetes que apresentam e em sua maior parte foi diagnosticada pela rede pública. O principal motivo da procura das redes particulares para a obtenção do medicamento é a ausência destes na Unidade Básica de Saúde. Além disso, a maior parte faz uso da medicação de primeira escolha, Metformina, casualmente fazem o teste de glicemia em jejum, e que raramente apresentaram alguma complicação do Diabetes Mellitus. A maioria dos pacientes entrevistados mudou o estilo de vida, principalmente em relação à alimentação. Conclui-se que se faz necessário uma estruturação na atenção básica municipal para melhor atender a demanda dos portadores do Diabetes Mellitus nas Unidades Básicas de Saúde a fim de minimizar as desigualdades no acesso às medicações no Brasil.

Palavras-chaves: Diabetes Mellitus, Política de Saúde, Farmácias.
\end{abstract}

Diabetes Mellitus is a chronic disease characterized by a glycemic imbalance resulting from the defect in the action / secretion of insulin. The objective of this study was to know the profile of Diabetes Mellitus patients who are looking for private drugstores to obtain medication and follow up of the disease. This study was carried out in two private pharmacies in the city of Itabaiana / Sergipe, in which a questionnaire with 10 questions about socioeconomic data and Diabetes Mellitus was applied. In total, 90 forms were analyzed from both private institutions, from which results were obtained as female predominance, low family income, with low level of schooling, who are not aware of the type of Diabetes they present and for the most part were diagnosed by public network. The main reason for the search of the private networks for obtaining the medicine is the absence of these in the Basic Health Unit. In addition, most use the medication of first choice, Metformin, casually do the fasting blood glucose test, but Rarely present any complications of Diabetes Mellitus. Most patients interviewed changed their lifestyle, especially in relation to diet. It is concluded that it is necessary a structuring in the municipal primary care to better meet the demand of patients with Diabetes Mellitus in the Basic Health Units in order to minimize the inequalities in access to medications in Brazil.

Keywords: Diabetes Mellitus, Health Policy, Pharmacies.

\section{INTRODUÇÃO}


O Diabetes mellitus (DM) além de ser considerada uma epidemia mundial se apresenta como um grande desafio para os serviços de saúde [1]. No Brasil, o DM tem uma alta incidência e é considerado um grande problema de saúde pública [2]. Atualmente o DM acomete uma população aproximada de 387 milhões de pessoas, e poderá alcançar 471 milhões em 2035. Os crescentes números podem estar relacionados ao crescimento e envelhecimento da população, ao sedentarismo, a obesidade e em especial ao aumento da expectativa de vida desses pacientes [3].

O DM é clinicamente observado a partir dos sintomas clássicos como polidipsia, poliúria, polifagia e emagrecimento, além das alterações na concentração de glicose no sangue [4]. Os valores de referência estabelecidos se enquadram em valores glicêmicos entre $\geq 126 \mathrm{mg} / \mathrm{dL}$ em jejum, $\geq 200 \mathrm{mg} / \mathrm{dL}$ na glicose plasmática casual e entre $\geq 140 \mathrm{a}<200 \mathrm{mg} / \mathrm{dL} 2$ horas após a administração de glicose por via oral. O DM é classificado em DM tipo 1, caracterizado pela destruição das células beta do pâncreas, DM tipo 2 que é definido como uma resistência a insulina e o DM gestacional que é uma intolerância a glicose durante o período gestacional [3].

Em 1986, com a VIII Conferência Nacional de Saúde foi fomentada a reestruturação do Sistema Nacional de Saúde implicando na criação Sistema Único de Saúde (SUS) em 1990, sendo o marco da reforma sanitária brasileira. Esta reforma foi embasada na Constituição Federal de 1988, com a universalidade, integralidade e equidade como diretrizes doutrinárias. A partir da elaboração das leis 8.080/90 e 8.142/90 foi garantida a descentralização dos serviços de saúde e as atribuições dos gestores nas três esferas do poder [5].

Nesta conjuntura, a Atenção Primária em Saúde se caracteriza como porta de entrada do usuário ao sistema e coordena o conjunto de respostas de acordo com as necessidades de saúde da população [6]. No concernente a linha de cuidados do DM se faz necessário à integralidade e a longitudinalidade dessa atenção. $\mathrm{O}$ acompanhamento inicial é feito pelo médico da atenção primária à saúde, identificando os fatores de risco, realizando orientações e o início da farmacoterapia, se necessário [1].

Legalmente, a portaria $\mathrm{n}^{\mathrm{o}} 2.583$ de 10 de outubro de 2007 estabelece todos os medicamentos que são disponibilizados pelo SUS para os portadores do DM. Além da lei no 11.347 de 27 de setembro de 2006, que dispõe sobre o fornecimento de medicamentos e materiais necessários à sua aplicação e monitoramento da glicemia capilar aos portadores do DM.

Apesar da existência legal e garantia da dispensação gratuita de medicamentos, os usuários do SUS ainda encontram dificuldades para conseguir gratuitamente os medicamentos receitados, pois segundo o Conselho Nacional de Secretários Municipais de Saúde, mais da metade dos brasileiros que precisou de medicamentos afirma não ter encontrado no SUS o remédio prescrito [7].

Neste sentido, pesquisar acerca do perfil dos portadores de DM em farmácias privadas pode esclarecer e demonstrar causas que evidenciem sua procura em redes particulares assim como a percepção dos mesmos sobre o acesso as unidades básicas de saúde de referência.

\section{MATERIAL E MÉTODOS}

Trata-se de um estudo descritivo, transversal e com abordagem quantitativa. Foi realizado em duas farmácias particulares no município de Itabaiana/Sergipe, o qual possui uma população estimada de 102.393 habitantes no ano de 2016, segundo o IBGE (Instituto Brasileiro de Geografia Estatística). A coleta dos dados foi realizada nos meses de janeiro, fevereiro e março de 2017 com disponibilidade do pesquisador nas instituições durante três dias na semana.

A amostra contou com uma seleção não probabilística e por conveniência e está concentrada nos pacientes diabéticos de ambos os gêneros e de todas as faixas etárias que comprem suas medicações para o DM nas referidas instituições e que aceitem participar da pesquisa assinando o termo de consentimento livre e esclarecido.

Para a obtenção dos dados foi aplicado um formulário com 10 questões objetivas obtendo dados socioeconômicos, os tipos de DM, local do diagnóstico, o tempo de início do diagnóstico, a resposta da busca pela farmácia particular para a compra dos medicamentos e quais são os mais utilizados, a frequência que realiza o teste de glicemia, complicações e mudanças no estilo de vida. 
Os dados foram armazenados e analisados no Microsoft Excel por meio da estatística descritiva. A pesquisa foi submetida e aprovada pelo comitê de ética com o respectivo número do parecer: 1.873.345, além de atender as normas e diretrizes da resolução nº 466/2012.

\section{RESULTADOS}

Foram analisados 90 formulários, dos quais 55 foram aplicados na farmácia 01 (área central da cidade) e 35 na farmácia 02 (periferia da cidade). Foi constatado que todos os entrevistados usavam ou já tinham usado o Sistema Único de Saúde em algum momento das suas vidas.

A tabela 1 mostra as características socioeconômicas dos entrevistados, no qual foi predominante o sexo feminino com $66,6 \%$, uma faixa etária de 60 anos ou mais $(63,3 \%)$, uma renda familiar de até dois salários mínimos $(61,1 \%)$ e uma baixa escolaridade $(38,3 \%)$.

Tabela 1:- Características socioeconômicas dos pacientes portadores de Diabetes Mellitus em farmácias particulares, Itabaiana/SE, 2017.

\begin{tabular}{lccc}
\hline \multicolumn{1}{c}{ Características Socioeconômicas } & $\begin{array}{c}\text { Farmácia 01 } \\
\mathbf{n}(\%)\end{array}$ & $\begin{array}{c}\text { Farmácia 02 } \\
\mathbf{n}(\%)\end{array}$ & $\begin{array}{c}\text { Geral } \\
\mathbf{n}(\%)\end{array}$ \\
\hline Sexo & $22(40,0 \%)$ & $8(28,8 \%)$ & $30(33,3 \%)$ \\
Masculino & $33(60,0 \%)$ & $27(77,1 \%)$ & $60(66,6 \%)$ \\
Feminino & & & \\
Faixa Etária & $22(40,0 \%)$ & $11(31,4 \%)$ & $33(36,6 \%)$ \\
$19-59$ anos & $33(60,0 \%)$ & $24(68,5 \%)$ & $57(63,3 \%)$ \\
60 anos ou mais & & & \\
Renda Familiar & $37(67,2 \%)$ & $18(51,4 \%)$ & $55(61,1 \%)$ \\
Até 2 salários mínimos & $13(23,6 \%)$ & $14(40,0 \%)$ & $27(30 \%)$ \\
$2-4$ salários mínimos & $3(5,4 \%)$ & $3(8,5 \%)$ & $6(6,6 \%)$ \\
$4-10$ salários mínimos & $2(3,6 \%)$ & - & $2(2,2 \%)$ \\
$10-20$ salários mínimos & & & \\
Escolaridade & $20(36,3 \%)$ & $15(42,8 \%)$ & $35(38,8 \%)$ \\
Analfabeto/Primário incompleto & $12(21,8 \%)$ & $9(25,7 \%)$ & $21(23,3 \%)$ \\
Primário completo/Ginasial incompleto & $6(10,9 \%)$ & $8(22,8 \%)$ & $14(15,5 \%)$ \\
Colegial incompleto & $1(1,8 \%)$ & $1(2,8 \%)$ & $2(2,2 \%)$ \\
Superior incompleto & $16(29,0 \%)$ & $2(5,71 \%)$ & $18(20,0 \%)$ \\
Superior Completo & & & \\
\hline
\end{tabular}

A tabela 2 expõe que dos pacientes entrevistados, 57,7\% não conhecem o seu tipo de DM, $68,8 \%$ foram diagnosticados na rede pública, $45,5 \%$ convive com a patologia de dois a cinco anos, 71 pessoas fazem uso da medicação de primeira escolha, metformina, $51,1 \%$ fazem ocasionalmente o teste de glicemia em jejum e 88,8\% não apresentaram complicações advindas do DM. 
Tabela 2: Distribuição do número de portadores de DM em relação ao tipo, a instituição de diagnóstico, tempo de diagnóstico, frequência da glicemia em jejum e a presença de complicações, Itabaiana/SE, 2017.

\begin{tabular}{|c|c|c|c|}
\hline Relação Paciente/Diabetes & $\begin{array}{c}\text { Farmácia } 01 \\
\text { n(\%) }\end{array}$ & $\begin{array}{c}\text { Farmácia } 02 \\
\text { n(\%) }\end{array}$ & $\begin{array}{l}\text { Geral } \\
\text { n(\%) }\end{array}$ \\
\hline \multicolumn{4}{|l|}{ Tipo de DM } \\
\hline Tipo 1 & $03(5,4 \%)$ & - & $03(3,3 \%)$ \\
\hline Tipo 2 & $24(43,6 \%)$ & $11(31,4 \%)$ & $35(38,8 \%)$ \\
\hline Não Sabe & $28(50,9 \%)$ & $24(68,5 \%)$ & $52(57,7 \%)$ \\
\hline \multicolumn{4}{|l|}{ Instituição de diagnóstico } \\
\hline Rede Pública & $35(63,6 \%)$ & $27(77,1 \%)$ & $62(68,8 \%)$ \\
\hline Rede Privada & $20(36,3 \%)$ & $08(22,8 \%)$ & $28(31,1 \%)$ \\
\hline \multicolumn{4}{|l|}{ Anos de diagnóstico do DM } \\
\hline Menos de 01 ano & $06(10,9 \%)$ & $02(5,7 \%)$ & $08(8,8 \%)$ \\
\hline $2-5$ anos & $23(41,8 \%)$ & $18(51,4 \%)$ & $41(45,5 \%)$ \\
\hline $6-10$ anos & $12(21,8 \%)$ & $11(31,4 \%)$ & $23(25,5 \%)$ \\
\hline Mais que 10 anos & $14(25,4 \%)$ & $04(11,4 \%)$ & $18(20 \%)$ \\
\hline \multicolumn{4}{|l|}{ Medicações em uso } \\
\hline Metformina & 43 & 28 & 71 \\
\hline Glibenclamida & 14 & 20 & 34 \\
\hline Glimepirida & 03 & 03 & 06 \\
\hline Insulina Regular & 05 & 05 & 10 \\
\hline Insulina NPH & 10 & 02 & 12 \\
\hline Outros & 04 & 01 & 05 \\
\hline \multicolumn{4}{|l|}{ Frequência da glicemia em jejum } \\
\hline Sempre & $13(23,6 \%)$ & $03(8,5 \%)$ & $16(17,7 \%)$ \\
\hline Às vezes & $27(49,0 \%)$ & $19(54,2 \%)$ & $46(51,1 \%)$ \\
\hline Raramente & $15(27,2 \%)$ & $13(37,1 \%)$ & $28(31,1 \%)$ \\
\hline \multicolumn{4}{|l|}{ Complicações do DM } \\
\hline Sim & $06(10,0 \%)$ & $04(11,4 \%)$ & $10(11,1 \%)$ \\
\hline Não & $49(89,0 \%)$ & $31(88,5 \%)$ & $80(88,8 \%)$ \\
\hline
\end{tabular}

A tabela 3 exibe que $65,5 \%$ do total dos pacientes procuram as redes particulares para a obtenção dos hipoglicemiantes orais devido ao mesmo estar em falta nas UBS (Unidade Básica de Saúde). Além disso, revelou que 88,8\% mudou o estilo de vida depois que foi diagnosticado com o DM e sendo a maior parte dessa mudança na alimentação. 
Tabela 3: Quantitativo do número de pacientes que buscam as farmácias particulares para obter os medicamentos do DM, mudança no estilo de vida e o modo dessa mudança, Itabaiana/SE, 2017.

Motivo da busca da medicação nas farmácias

particulares, mudança e modo da mudança Farmácia 01 Farmácia 02 Geral

$\begin{array}{llll}\text { no estilo de vida } & \mathbf{n}(\%) & \mathbf{n}(\%) & \mathbf{n}(\%)\end{array}$

\begin{tabular}{lccc}
\hline Motivo da busca & & & \\
Não ter disponibilidade na UBS & $36(65,4 \%)$ & $23(65,7 \%)$ & $59(65,5 \%)$ \\
Preferência pela rede particular & $13(23,6 \%)$ & $06(17,1 \%)$ & $19(21,1 \%)$ \\
A farmácia fica próxima da residência & $05(9,0 \%)$ & $06(17,1 \%)$ & $11(12,2 \%)$ \\
Confia na rede particular & $01(1,8 \%)$ & - & $01(1,1 \%)$ \\
Mudança no estilo de vida & & & \\
Sim & $48(87,2 \%)$ & $32(91,4 \%)$ & $80(88,8 \%)$ \\
Não & $07(12,7 \%)$ & $03(8,5 \%)$ & $10(11,1 \%)$ \\
Modo da mudança no estilo de vida & & & \\
Alimentação & 47 & 31 & $78 \%$ \\
Exercício Físico & 10 & 06 & $16 \%$ \\
\hline
\end{tabular}

\section{DISCUSSÃO}

A pesquisa evidenciou que as mulheres procuram mais os serviços de saúde a fim de encontrar medicações para o DM, tal fato pode implicar em maior preocupação do sexo feminino com relação à sua saúde, quando comparado aos homens. Estudos nacionais corroboram com os resultados encontrados $[8,9]$.

De acordo com o censo populacional de 2010 do IBGE, o percentual de pessoas idosas passou de $8,57 \%$ para $11,16 \%$. A transição no perfil etário, de uma população jovem para uma população mais envelhecida, está resultando em alterações no perfil epidemiológico. As doenças infectocontagiosas diminuem sua incidência nos jovens, enquanto que as doenças crônicas não transmissíveis típicas do envelhecimento a exemplo o DM aumenta na população idosa. Essa informação foi comprovada na presente pesquisa e em outros estudos nacionais que confirmam que o DM é prevalente de uma população idosa [10,11].

Verificou-se uma predominância de idosos com baixo nível de escolaridade e pouca renda familiar. Dados equivalentes foram encontrados em alguns trabalhos no Brasil [12,13,14]. A baixa escolaridade influencia na implementação de programas de educação em DM, visto que pode dificultar o entendimento das orientações terapêuticas e médica assim como no ensino de novos hábitos de vida que melhorem a qualidade de vida [15].

No estudo houve uma observância prevalente de pacientes que não conheciam o tipo de DM que portavam, fato que evidencia o desconhecimento da população sobre a doença e consequentemente sobre sua saúde, como encontrado também pelo estudo de Marques, et al (2012) [16] que retrata 50\% dos portadores de DM não sabem seu diagnóstico correto. Segundo Oliveira e Vencio (2016) [3], o DM tipo 2 é o mais frequente entre a população brasileira. E foi através da observação cautelosa dos participantes, somado aos tipos de medicamentos em uso, que concluímos sobre a grande parte dos participantes que desconheciam o seu diagnóstico de portadores do DM 2. Esse fato pode estar associado ao nível de escolaridade dos pacientes que influencia no conhecimento dos mesmos a cerca do DM [17].

Quanto ao motivo da busca dos medicamentos pelos portadores do DM em farmácias particulares foi verificado que a maioria $(65,5 \%)$ dos pacientes justificam a compra das suas medicações por falta nas UBS. Segundo o estudo de Sartor e Freitas (2014) [18], a qualificação constante dos serviços de saúde é extremamente necessária para a concretização dos princípios e 
diretrizes do SUS fato que pode contribuir ativamente para suprir a demanda no concernente à disponibilização de medicações. Pesquisa realizada por Boing et al. (2013) [19] também sinaliza que a disponibilização das medicações de acordo com a necessidade dos usuários, é ainda um grande desafio. Mesmo com a criação da Política Nacional de Medicamentos, Política Nacional de Assistência Farmacêutica e o Programa Farmácia Popular que garantem a disponibilização gratuita de medicações, ainda existem lacunas a serem melhoradas para uma distribuição equânime para com os usuários do Sistema Único de Saúde.

Ademais, o hábito de uma vida saudável no controle do tratamento do DM é de extrema importância para melhoria da condição de vida dos pacientes [20]. A pesquisa mostrou que $88,8 \%$ dos pesquisados optaram pela mudança no estilo de vida após o diagnóstico com o DM, especialmente no tocante a alimentação. Em seu estudo, Antão et al. (2013) [21] mostraram que uma alimentação inadequada está relacionada diretamente com as complicações do DM, fato corroborado na presente pesquisa quando evidencia mudanças no estilo de vida e apresenta $11,1 \%$ dos pesquisados com alguma complicação do DM.

As complicações do DM são as principais causas das morbimortalidades dos pacientes diabéticos [22], porém o resultado da presente pesquisa identificou que apenas $11,1 \%$ dos pacientes tiveram alguma complicação em decorrência da doença.

\section{CONCLUSÃO}

Concluiu-se que o perfil dos portadores de DM que procuram as farmácias particulares para adquirir suas medicações caracteriza-se prioritariamente por população idosa feminina, com um baixo nível de escolaridade, que desconhecem o tipo de DM o qual foi diagnosticado. A maioria mudou o estilo de vida para conviver melhor com a doença além de projetar uma expectativa de vida ainda maior. O principal motivo da busca de medicamentos em farmácias particulares devese ao motivo da ausência dos mesmos nas UBS.

A garantia de acesso aos medicamentos no Brasil dentro do SUS, ainda apresenta lacunas e fragilidades para atender a demanda de usuários necessitados. Desta forma é de suma importância elaborar e intensificar as estratégias de acesso aos fármacos no país, assim como uma melhora do monitoramento para diminuir e/ou eliminar as desigualdades no controle e tratamento do agravo.

\section{REFERÊNCIAS BIBLIOGRÁFICAS}

1. Brasil. Ministério da Saúde. Secretaria de Atenção à Saúde. Departamento de Atenção Básica. Estratégias para o cuidado da pessoa com doença crônica: diabetes mellitus / Ministério da Saúde, Secretaria de Atenção à Saúde, Departamento de Atenção Básica. - Brasília: Ministério da Saúde, 2013. 160 p.: il. (Cadernos de Atenção Básica, n. 36).

2. Petermann XB, Machado IS, Pimentel BN, Miolo SB, Martins LR, Fedosse E. Epidemiologia e cuidado à Diabetes Mellitus praticado na Atenção Primária à Saúde: uma revisão narrativa. Rev Saúde. 2015 Jan/Jul;41(1):49-56.

3. Diretrizes da Sociedade Brasileira de Diabetes (2015-2016) / Adolfo Milech...[et. al.]; organização José Egidio Paulo de Oliveira, Sérgio Vencio - São Paulo: A.C. Farmacêutica; 2016.

4. Motta VT. Bioquímica clínica para laboratório: princípios e interpretações. 5 ed. Rio de Janeiro: Ed. Medbook; 2009. 400 p.

5. Guidoni CM, Oliveira CMX, Freitas O, Pereira LRL. Braz. Assintência ao diabeters no Sistema Único de Saúde: análise do modelo atual. Braz. J. Pharm. Sci. 2009 Jan/Mar;45(1), doi:10.1590/S198482502009000100005

6. Brasil. Ministério da Saúde. Secretaria de Atenção à Saúde. Departamento de Atenção Básica. Estratégias para o cuidado da pessoa com doença crônica / Ministério da Saúde, Secretaria de Atenção à Saúde, Departamento de Atenção Básica. - Brasília: Ministério da Saúde, 2014. 162 p.: il. (Cadernos de Atenção Básica, n. 35)

7. Salazar AL, Grou KB, Sampaio L, Scheffer M. Medicamento um direito essencial. Disponível em: http://www.dhnet.org.br/dados/cartilhas/a_pdf/114_cartilha_direito_medicamentos.pdf. Acesso em: 24/04/2017, às 13:08horas.

8. Costa-Junior FM, Maia ACB. Concepções de Homens Hospitalizados sobre a Relação entre Gênero e Saúde. Psicologia: Teoria e Pesquisa. 2009 Jan-Mar;25(1):55-63. 
9. Alves RF, Silva, RP, Ernesto MV, Lima AGB, Souza FM. Gênero e saúde: o cuidar do homem em debate. Psicologia: Teoria e prática. 2011 Dez;25(3):152-166.

10. Ramos RSPS. Diabetes e fatores associados em idosos assistidos em serviço geronto-geriátrico. [Dissertação]. Recife: Universidade Federal de Pernambuco; 2012. 111 p.

11. Ramos RSPS, Alborghetti KT, Oliveira MAS, Silveiro MR. Diabetes mellitus tipo II em pessoas idosas: estratégias utilizadas pelos usuários da rede pública de saúde em um município do Sul de Santa Catarina. BCEH. 2012 Mai/Ago;9(2):200-212.

12. Silva, RCP, Simões, MJS, Leite, AA. Fatores de risco para doenças cardiovasculares em idosos com diabetes mellitus tipo 2. Rev Ciên Farm Básica Apl. 2007 Ago;28(1):113-121.

13. Stopa SR, César CLG, Segri NJ, Goudbaum M, Guimarães VMV, Alves MCGP, et al. Diabetes autorreferido em idosos: comparação das prevalências e medidas de controle. Rev Saúde Pública. 2014;48(4):554-562, doi:10.1590/S0034-8910.2014048005219

14. Morais GFC, Soares MJGO, Costa MML, Santos IBC. Morais GFC, et al. Conhecimento e práticas dos diabéticos acerca das medidas preventivas para lesões de membros inferiores. Rev Baiana, 2009 $\mathrm{Jul} / \mathrm{Set} ; 33(3): 361-371$.

15. Modeneze, DM. Qualidade de vida e diabetes: limitações físicas e culturais de um grupo específico. [Dissertação]. Campinas/SP. UNICAMP; 2004. 98p.

16. Marques, HS, Souza, LP, Domingos, NAM. Diabetes Tipo II: qualidade de vida e aspectos psicológicos. Arq Ciênc Saúde, 2012. Abr/Jun;19(2):16-22.

17. Rodrigues FFLR, Santos MA, Teixeira CRS, Gonela JT, Zanetti ML. Relação entre conhecimento, atitude, escolaridade e tempo de doença em indivíduos com diabetes mellitus. Acta Paul Enferm, 2012. Outubro; 25(2):284-290, doi:10.1590/S0103-21002012000200020

18. Sartor BN, Freitas SFT. Modelo para avaliação do serviço de dispensação de medicamentos na atenção básica à saúde. Rev Saúde Pública, 2014. Maio;48(5):827-836, doi: 10.1590/S00348910.2014048005135.

19. Boing AG, Bertoldi AD, Boing AF, Bastos JL, Peres KG. Acesso a medicamentos no setor público: análise de usuários do Sistema Único de Saúde no Brasil. Card. Saúde Pública, 2013. Abril;29(4):691701.

20. Melo SM. Mudanças de estilo de vida em portadores de diabetes mellitus tipo 2 na estratégia de saúde da familia de burutis das mulatas. Várzea da palma/MG. [Tese]. Universidade Federal de Minas Gerais; 2015. 95p.

21. Antão JYFL. Complicações do diabetes mellitus: uma reflexão acerca da atuação do enfermeiro. eciência, 2013. Outubro;1(1).

22. Pasqualotto KR, Alberton D, Frigeri HR. Diabetes mellitus e Complicações. J. Biotec. Biodivers, 2012. Nov;3(4):134-145. 